\title{
Analysis of plastic zones in surrounding rocks around a circular tunnel considering the effect of intermediate principal stress and heterogeneity
}

\author{
Shuxin Deng ${ }^{1}$, Yonglai Zheng ${ }^{2, *}$, Lipo Feng ${ }^{3}$, Le Van Tuan ${ }^{2}$, Cuizhou Yue ${ }^{2}$, and Zhuorui $\mathrm{Wu}^{2}$,* \\ ${ }^{1}$ Nanjing University of Science and Technology, School of Mechanical Engineering, 210094 Nanjing, China \\ ${ }^{2}$ Tongji University, College of Civil Engineering, 200092 Shanghai, China \\ ${ }^{3}$ Jinhua Rail Transit Investment and Construction Co., Ltd., 321017 Jinhua Zhejiang, China
}

\begin{abstract}
Based on a modified Mohr-Coulomb criterion with a non-uniform coefficient, a calculation method of plastic zone boundary of surrounding rocks in a circular tunnel in non-uniform stress field is established. Both the effects of intermediate principal stress and heterogeneity are studied. With the increase of the intermediate principal stress, the plastic zone size of the surrounding rocks will decrease first and then increase. Lateral pressure coefficient has an effect on the shape of the plastic zone. With the increase of lateral pressure coefficient, the plastic zone gradually becomes uniform, and the failure of surrounding rock develops upward and downward from both sides. As non-uniform coefficient increases, the material is more uniform and the effect of intermediate principal stress on the plastic zone is less significant. If the effect of intermediate principal stress is not taken into account, the calculation results tend to be consistent with results calculated by the Mohr-Coulomb criterion, which are considered to be conservative.
\end{abstract}

\section{Introduction}

In underground engineering, the stability of surrounding rocks is a significant research topic, which is related to the security of tunnels during construction and operation. Under excavation unloading, surrounding rocks remain purely elastic until the failure criterion is satisfied. As the excavation continues, surrounding rocks around the tunnel become more and more plasticized, forming an increasingly thick plastic zone [1]. The size of the plastic zone is an important basis for the stability analysis of surrounding rock and the quantitative design of tunnel support.

To obtain the plastic zone of the surrounding rocks, the stress distribution of the surrounding rock should be first determined. For a circular tunnel, if the surrounding rocks are assumed to be a homogeneous medium, the stress distribution of the surrounding rock can be determined by an analytical solution $[2,3]$. Besides the theoretical analysis, numerical simulation [4, 5] or physical experiments $[6,7]$ can be applied to study the stress distribution and failure behavior of surrounding rocks in more complex cases. Jiang et al. [8] developed a theoretical method to predict the generation of plastic zones around tunnels in soft rocks and presented a judgment method to assess the extent of rock failure. Brown et al. [9] presented a method to calculate the response curve for rock mass initially subjected to a hydrostatic in-situ stress field, using the nonlinear peak and residual rock mass strength criteria. Ogawa and Lo [10] developed analytical elastoplastic solutions for stresses and displacements around a circular opening based on the incorporation of a parabolic and a linear Mohr-Coulomb yield criterion.

In general, the rocks are in a polyaxial stress state [11], as plotted in Fig.1. $\sigma_{1}$ is the maximum principal (tangential) stress, $\sigma_{3}$ is the minimum principal (radial) stress, and $\sigma_{2}$ is the intermediate principal stress, which is often equal to the in-situ stress along the tunnel axis. Previous studies [11-15] have clearly shown that the intermediate principal stress has an effect on rock strength and deformation. When using Hoek-Brown criterion [9] or Mohr-Coulomb criterion [16] for elasticplastic analysis of surrounding rocks, the effect of intermediate principal stress is not taken into account, which obviously does not reflect the real engineering conditions. Other strength criteria, like Drucker-Prager criterion [17] and 3D Griffith criterion [18], are considered to have overestimated the effect of intermediate principal stress [14]. Because these strength criteria cannot rightly reflect the mechanism of the intermediate principal stress effect. The authors' previous studies $[19,20]$ have shown that the intermediate principal stress effect can be regarded as a resultant effect of the meso-heterogeneity and macroscopic non-uniform stress field. Based on such a consideration, by modifying the Mohr-Coulomb criterion with a non-uniform coefficient, a strength criterion is proposed to quantitatively reflect the intermediate principal stress effect on rock strength.

\footnotetext{
* Corresponding author: (Yonglai Zheng)zyll@tongji.edu.cn; (Zhuorui Wu)wzr341@163.com
} 
In the present study, a calculation method is developed to analysis the plastic zones in surrounding rocks around a circular tunnel, based on the modified Mohr-Coulomb criterion with a non-uniform coefficient. In this way, both effects of intermediate principal stress and heterogeneity are considered.

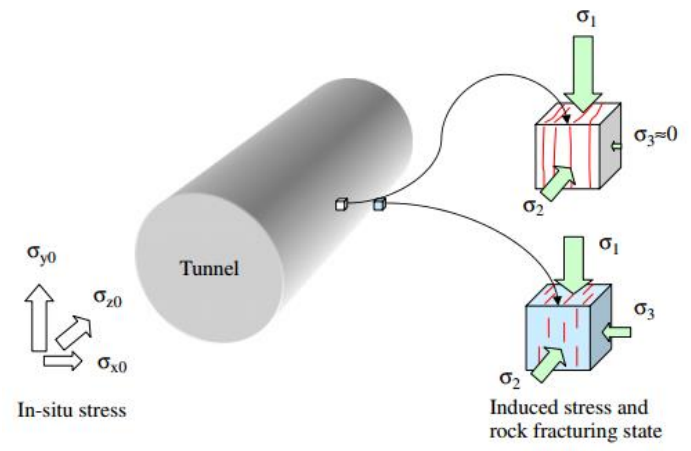

Fig. 1. Stress states of surrounding rocks [11]

\section{Stress field in surrounding rocks around a circular tunnel}

The surrounding rocks are always treated as a crosssection of a cylindrical cavity under plane - strain conditions and the failure behavior of the surrounding rocks can be easily analyzed in the two-dimensional case. When buried depth $Z$ is more than or equal to 20 times of tunnel radius $a$, the gravity of rocks in influence zone ( 3 to 5 times of the tunnel radius $a$ ) can be ignored. In this case, horizontal rock stress can be simplified as uniformly distributed loadings, and the problem becomes a plane-strain circular hole problem in which both the stress and structure are axisymmetric. The analytical expressions of the tangential and radial stresses of the surrounding rocks can be expressed as $[2$, 3]:

$$
\left.\left.\begin{array}{rl}
\sigma_{r}=\sigma_{0}\left(1-\frac{a^{2}}{r^{2}}\right)+p_{0} \frac{a^{2}}{r^{2}} \\
\sigma_{\theta}=\sigma_{0}\left(1+\frac{a^{2}}{r^{2}}\right)-p_{0} \frac{a^{2}}{r^{2}}
\end{array}\right\} \begin{array}{rl}
(1) \\
\sigma_{r}=\frac{1}{2}(1-\lambda) \sigma_{0}\left(1-\frac{a^{2}}{r^{2}}\right)-\frac{1}{2}(1-\lambda) \sigma_{0}\left(1-4 \frac{a^{2}}{r^{2}}+3 \frac{a^{4}}{r^{4}}\right) \cos 2 \theta \\
\sigma_{\theta}=\frac{1}{2}(1-\lambda) \sigma_{0}\left(1+\frac{a^{2}}{r^{2}}\right)+\frac{1}{2}(1-\lambda) \sigma_{0}\left(1+3 \frac{a^{4}}{r^{4}}\right) \cos 2 \theta \\
\tau_{r \theta}=\frac{1}{2}(1-\lambda) \sigma_{0}\left(1+2 \frac{a^{2}}{r^{2}}-3 \frac{a^{4}}{r^{4}}\right) \sin 2 \theta
\end{array}\right\}
$$

where $\sigma_{r}$ and $\sigma_{c}$ are the radial stress and tangential stress at a certain point in polar coordinates, respectively; $\sigma_{c}$ is the in-situ stress; $r$ is the polar radius; $a$ is the tunnel radius; $p_{0}$ is the supporting pressure applied on the inner boundary of the tunnel.

Considering a more general situation, it is assumed that the horizontal stress of the deep buried circular tunnel is an axial symmetry regarding the vertical axis and the vertical stress is an axial symmetry regarding the horizontal axis. The vertical stress and the horizontal stress are assumed to be not equal, denoted as $\sigma_{0}$ and $\lambda \sigma_{0}$, respectively, where $\lambda$ is the lateral pressure coefficient. As shown in Fig.2, this problem can be solved by the superposition principle [21]. The stress state of a circular tunnel under non-uniform stress field can be divided into two cases: uniform stress field (case I) and unidirectional compressive stress field (case II).

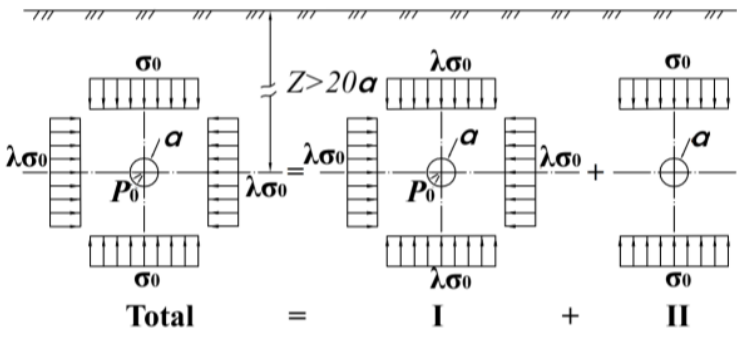

Fig. 2. Simplified stress diagram of the deeply buried tunnel in the non-uniform stress field

Equation (1) is the solution for case I. For case II, the analytical solution of the stress field under unidirectional compressive stress $(1-\lambda) \sigma_{0}$ is:

The expression of non-uniform stress field can be obtained by superposition of case I and case II as follows:

$$
\left.\begin{array}{l}
\sigma_{r}=\frac{1}{2}(1+\lambda) \sigma_{0}\left(1-\frac{a^{2}}{r^{2}}\right)-\frac{1}{2}(1-\lambda) \sigma_{0}\left(1-4 \frac{a^{2}}{r^{2}}+3 \frac{a^{4}}{r^{4}}\right) \cos 2 \theta+p_{0} \frac{a^{2}}{r^{2}} \\
\sigma_{\theta}=\frac{1}{2}(1+\lambda) \sigma_{0}\left(1+\frac{a^{2}}{r^{2}}\right)+\frac{1}{2}(1-\lambda) \sigma_{0}\left(1+3 \frac{a^{4}}{r^{4}}\right) \cos 2 \theta-p_{0} \frac{a^{2}}{r^{2}} \\
\tau_{r \theta}=\frac{1}{2}(1-\lambda) \sigma_{0}\left(1+2 \frac{a^{2}}{r^{2}}-3 \frac{a^{4}}{r^{4}}\right) \sin 2 \theta
\end{array}\right\}
$$




\section{Plastic zone in surrounding rocks around a circular tunnel}

\subsection{Strength criterion}

Under excavation unloading, surrounding rocks remain elastic until the strength criterion is satisfied. As mentioned above, Mohr-Coulomb criterion, HoekBrown criterion, Drucker-Prager criterion, and 3D Griffith criterion ignore or overestimate the effect of the

intermediate principal stress. Furthermore, these criteria do not consider material heterogeneities, regarded as the root cause of the intermediate principal stress effect [20]. Therefore, we use the modified Mohr-Coulomb criterion with a non-uniform coefficient to calculate the plastic zone boundary. The modified Mohr-Coulomb criterion can be expressed as:

$$
\tau_{13}=f_{1}\left(\sigma_{3}\right) \cdot f_{2}\left(\mu_{\sigma}\right)
$$

where

$$
\left.\begin{array}{l}
\tau_{13}=\frac{\sigma_{1}-\sigma_{3}}{2} \\
f_{1}\left(\sigma_{3}\right)=\frac{\sigma_{3} \sin \phi+c \cos \phi}{1-\sin \phi} \\
f_{2}\left(\mu_{\sigma}\right)=\left[\frac{2 \pi \int_{0}^{\pi / 2} \sin ^{m}(2 \alpha) \mathrm{d} \alpha}{\int_{0}^{2 \pi} \int_{0}^{\pi / 2} f_{3}^{m}\left(\varphi, \alpha, \mu_{\sigma}\right) \mathrm{d} \alpha \mathrm{d} \varphi}\right]^{1 / \mathrm{m}} \\
f_{3}\left(\varphi, \alpha, \mu_{\sigma}\right)=2 \sqrt{\sin ^{2} \alpha\left[\frac{\left(1-\mu_{\sigma}\right)^{2}}{4} \cos ^{2} \varphi+\sin ^{2} \varphi\right]-\sin ^{4} \alpha\left[\frac{1-\mu_{\sigma}}{2} \cos ^{2} \varphi+\sin ^{2} \varphi\right]^{2}} \\
\mu_{\sigma}=\frac{2 \sigma_{2}-\sigma_{1}-\sigma_{3}}{\sigma_{1}-\sigma_{3}}
\end{array}\right\}
$$

where $\mu_{\sigma}$ is the Lode parameter, which is used to distinguish between the different shear stress states in three dimensions; $\alpha$ and $\varphi$ are azimuth angles; $c$ is the cohesive strength; $\phi$ is the internal friction angle; $m$ is shape parameter of Weibull distribution and can be considered as the non-uniform coefficient.

When $m \rightarrow \infty, f_{2}\left(\mu_{\sigma}\right)=1$, equation (4) becomes $\tau_{13}=f_{1}\left(\sigma_{3}\right)$, which is equivalent to Mohr-Coulomb criterion. That means if materials are absolutely homogeneous, there is no intermediate principal stress effect. Equation (4) matches experimental results and numerical simulation results better than common strength criteria [19]. Furthermore, both effects of the meso-heterogeneity and macroscopic non-uniform stress field are taken into account in the modified MohrCoulomb criterion.

\subsection{Calculation method of the plastic zone boundary}

Under plane-strain conditions, we have [22]

$$
\left.\begin{array}{l}
\sigma_{1}=\sigma_{\max }=\frac{1}{2}\left(\sigma_{r}+\sigma_{\theta}\right)+\sqrt{\left(\frac{\sigma_{r}-\sigma_{\theta}}{2}\right)^{2}+\tau_{r \theta}{ }^{2}} \\
\sigma_{3}=\sigma_{\min }=\frac{1}{2}\left(\sigma_{r}+\sigma_{\theta}\right)-\sqrt{\left(\frac{\sigma_{r}-\sigma_{\theta}}{2}\right)^{2}+\tau_{r \theta}{ }^{2}}
\end{array}\right\}
$$

Substituting equation (6) into equation (4), the failure conditions can be expressed as

$$
\sqrt{\left(\frac{\sigma_{r}-\sigma_{\theta}}{2}\right)^{2}+\tau_{r \theta}^{2}}=\frac{\left(\sigma_{r}+\sigma_{\theta}\right) \sin \varphi+2 c \cos \varphi}{2\left(\frac{1-\sin \varphi}{f_{2}\left(\mu_{\sigma}\right)}+\sin \varphi\right)}
$$

Substituting equation (3) into equation (7) and assuming $k=a^{2} / r^{2}$, the boundary line expression of the plastic zone can be obtained as follows

$$
f(k)=a_{0}+a_{1} k+a_{2} k^{2}+a_{3} k^{3}+a_{4} k^{4}=0
$$




$$
\begin{aligned}
& a_{0}=(1-\lambda)^{2}-\frac{\sin ^{2} \phi}{\left[\frac{1-\sin \phi}{f_{2}\left(\mu_{\sigma}\right)}+\sin \phi\right]^{2}}\left(1+\lambda+\frac{2 c}{\sigma_{0} \tan \phi}\right)^{2} \\
& a_{1}=-4(1-\lambda)^{2} \cos 4 \theta-2(1-\lambda) \cos 2 \theta\left\{\left(\frac{2 p_{0}}{\sigma_{0}}-1-\lambda\right)+\frac{2 \sin ^{2} \phi}{\left[\frac{1-\sin \phi}{f_{2}\left(\mu_{\sigma}\right)}+\sin \phi\right]^{2}}\left(1+\lambda+\frac{2 c}{\sigma_{0} \tan \phi}\right)\right\} \\
& a_{2}=\left(\frac{2 p_{0}}{\sigma_{0}}-1-\lambda\right)^{2}-2(1-\lambda)^{2}+4(1-\lambda)\left(\frac{2 p_{0}}{\sigma_{0}}-1-\lambda\right) \cos 2 \theta+4(1-\lambda)^{2}\left(3-\frac{\sin ^{2} \phi}{\left[\frac{1-\sin \phi}{f_{2}\left(\mu_{\sigma}\right)}+\sin \phi\right]^{2}}\right) \cos ^{2} 2 \theta \\
& a_{3}=-6(1-\lambda)\left[2(1-\lambda)+\left(\frac{2 p_{0}}{\sigma_{0}}-1-\lambda\right) \cos 2 \theta\right] \\
& a_{4}=9(1-\lambda)^{2}
\end{aligned}
$$

Equation (8) is the boundary line expression of the plastic zone, which can be solved by Newton iteration method. In the uniformly distributed stress field (i.e., $\lambda=1$ ), from equation (8), plastic zone radius can be obtained as follows:

$$
r_{p}=a \sqrt{\frac{1-\frac{p_{0}}{\sigma_{0}}}{\left(1+\frac{c}{\sigma_{0} \tan \phi}\right) \frac{\sin \phi f_{2}\left(\mu_{\sigma}\right)}{1-\sin \phi+\sin \phi f_{2}\left(\mu_{\sigma}\right)}}}
$$

\section{Examples and results}

\subsection{Calculation parameters}

For the convenience of comparison, referring to the parameter values in literature [23], we have: the in-situ stress $\sigma_{0}=40 \mathrm{MPa}$; shear strength parameter $c=2.9 \mathrm{MPa}, \phi=30^{\circ}, m=3$; lateral pressure coefficients $\lambda=0.3,0.5,0.8 ;$ supporting pressure $p_{0}=0$. According to equation (8), the boundary lines of the plastic zone of surrounding rock in non-uniform stress field are obtained by programming with MATLAB. Due to axisymmetry, only a quarter of the boundary lines are potted, as shown in Fig. 3.

\subsection{Effect of intermediate principal stress}

From Fig. 3, calculation results can well reflect the effect of intermediate principal stress on the plastic zone boundary. When $\mu_{\sigma}=-1 \quad\left(\sigma_{2}=\sigma_{3}\right)$, the calculation result of equation (8) in the present paper is consistent with the calculation result based on the unified strength criterion [23]. When $\mu_{\sigma}>0$, the range of plastic zone calculated by equation (8) increased with the increase of $\mu_{\sigma}$, which was caused by the interval effect of intermediate principal stress. As $\sigma_{2}$ increases form $\sigma_{2}=\sigma_{3}$ (i.e., $\mu_{\sigma}=-1$ ) to $\sigma_{2}=\sigma_{1}$ (i.e., $\mu_{\sigma}=1$ ), rock strength has an initial increase and subsequent decrease. According to this result, the boundary of the plastic zone will decrease first and then increase. The calculation results in literature [23] do not reflect this phenomenon.

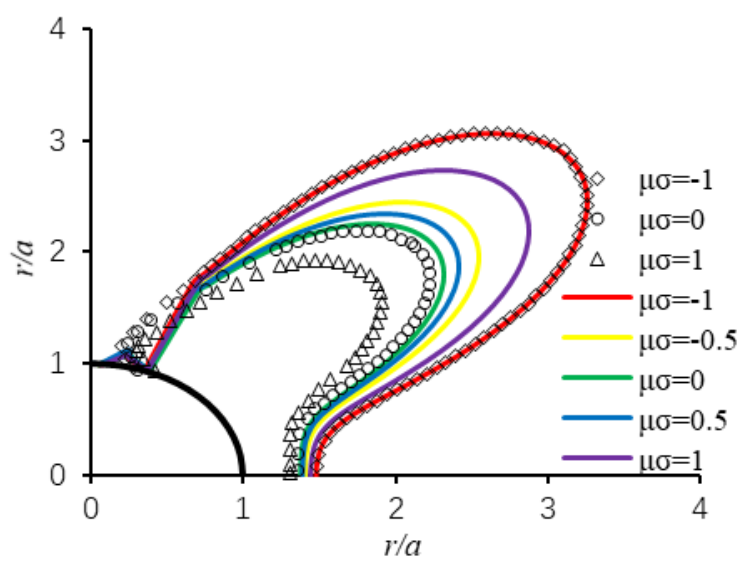

(a) $\lambda=0.3$

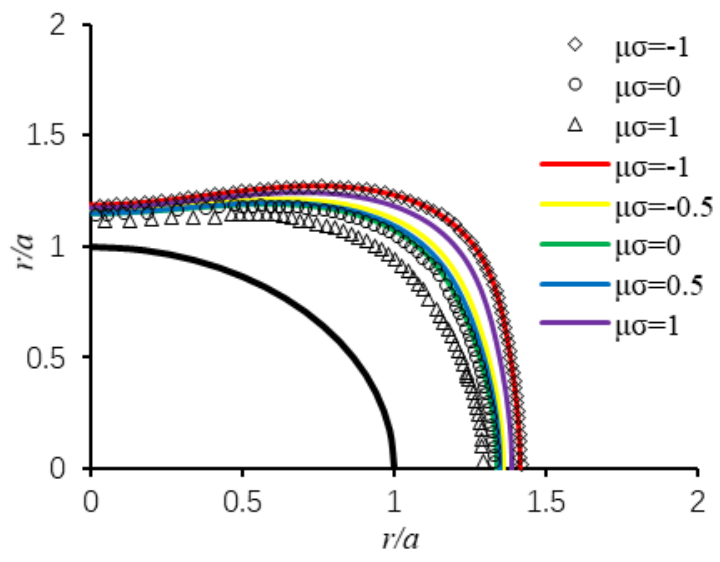

(b) $\lambda=0.5$ 


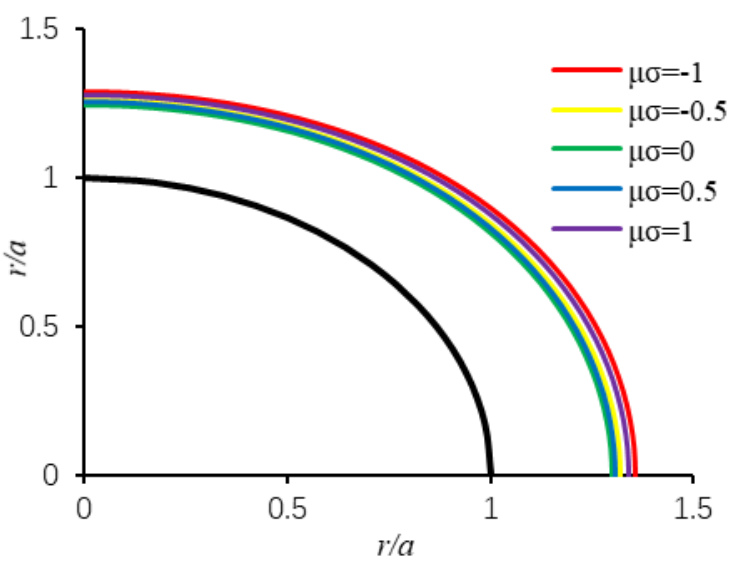

(c) $\lambda=0.8$

Fig. 3. Plastic zone boundary of surrounding rocks under non-uniform stress field. Diamond, circular, and triangle symbols are the calculation results of literature [23]; color solid lines are calculation results of equation (8); the black solid line is the tunnel boundary.

\subsection{Effect of lateral pressure coefficient}

As shown in Fig. 3 , when $\lambda$ is small, the distribution of the plastic zone is extremely uneven, and the failure of the surrounding rock occurs on both sides of the tunnel. As $\lambda$ increases, the distribution of plastic zone gradually becomes uniform, and the top and bottom of surrounding rocks will also collapse. When $\lambda$ is close to 1 , the shape of the plastic zone is close to a circle. These results are consistent with the field observations, and also with the numerical results [23].

\subsection{Effect of the non-uniform coefficient}

Calculation results with different non-uniform coefficients are shown in Fig. 4. As $m$ increases, the material is more uniform and the effect of intermediate principal stress is less significant. There will be a larger plastic zone with a large $m$, because the peak of rock strength becomes smaller. When $m \rightarrow \infty$, intermediate principal stress has no effect on plastic zone size. In this case, the calculation results of the plastic zone are consistent with the results calculated by the MohrCoulomb criterion, which are considered to be conservative.

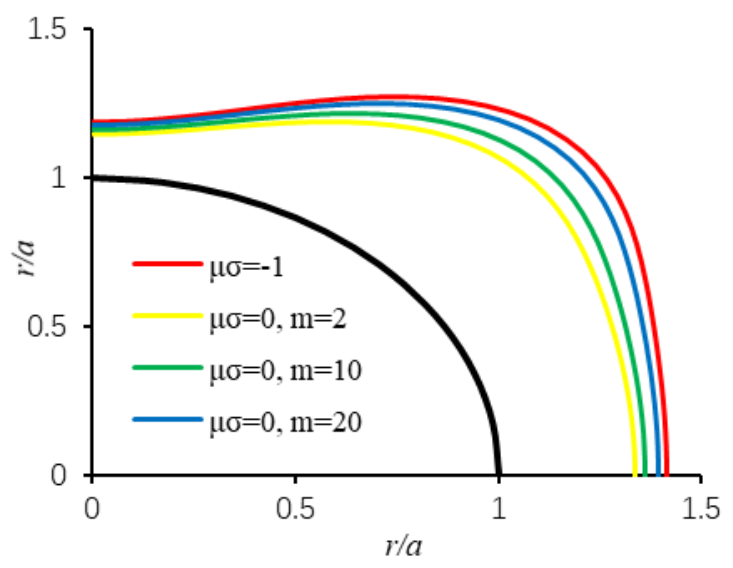

Fig. 4. Effect of non-uniform coefficients on plastic zone boundary in surrounding rocks around a circular tunnel. Colour solid lines are calculation results of equation (8); the black solid line is the tunnel boundary.

\section{Conclusions}

Based on the modified Mohr-Coulomb criterion with a non-uniform coefficient, calculation method of plastic zone boundary of surrounding rocks in a circular tunnel in non-uniform stress field is established. The effect of intermediate principal stress, lateral pressure coefficient, and heterogeneity are studied.

The results show that the interval effect of intermediate principal stress on rock strength is also reflected in the effect of the plastic zone size of the surrounding rocks. With the increase of the intermediate principal stress, the plastic zone size of the surrounding rocks will decrease first and then increase. Lateral pressure coefficient has an effect on the shape of the plastic zone. With the increase of lateral pressure coefficient, the plastic zone gradually becomes uniform from non-uniformity, and the failure of surrounding rock develops upward and downward from both sides, which is consistent with the on-site observations. As nonuniform coefficient increases, the material is more uniform and the effect of intermediate principal stress on the plastic zone is less significant. If the effect of intermediate principal stress is not taken into account, the calculation results tend to be conservative, which will not be conducive to obtaining greater economic benefits.

\section{References}

1. Vrakas, A. \& Anagnostou, G. A finite strain closedform solution for the elastoplastic ground response curve in tunnelling. Int. J. Numer. Anal. Methods Geomech. 38, 1131-1148 (2014).

2. Jaeger, C. Rock mechanics and engineering. (Cambridge University Press, 1979).

3. Brady, B. H. \& Brown, E. T. Rock mechanics: for underground mining. (Springer science \& business media, 2013). 
4. Huang, Z., Tang, C. \& Cai, M. Numerical Analysis of Unloading-Induced Rock Failure: Insight into Strainburst Mechanism. Indian Geotech. J. 48, 558563 (2018).

5. Lisjak, A., Garitte, B., Grasselli, G., Müller, H. R. \& Vietor, T. The excavation of a circular tunnel in a bedded argillaceous rock (Opalinus Clay): Shortterm rock mass response and FDEM numerical analysis. Tunn. Undergr. Space Technol. 45, 227248 (2015).

6. Lin, P., Liu, H. \& Zhou, W. Experimental study on failure behaviour of deep tunnels under high in-situ stresses. Tunn. Undergr. Space Technol. 46, 28-45 (2015).

7. Jiang Mingjing et al. Experimental Investigation of the Deformation Characteristics of Natural Loess under the Stress Paths in Shield Tunnel Excavation. Int. J. Geomech. 17, 04017079 (2017).

8. Jiang, Y., Yoneda, H. \& Tanabashi, Y. Theoretical estimation of loosening pressure on tunnels in soft rocks. Tunn. Undergr. Space Technol. 16, 99-105 (2001).

9. Brown Edwin T., Bray John W., Ladanyi Branko \& Hoek Evert. Ground Response Curves for Rock Tunnels. J. Geotech. Eng. 109, 15-39 (1983).

10. Ogawa, T. \& Lo, K. Y. Effects of dilatancy and yield criteria on displacements around tunnels. Can. Geotech. J. 24, 100-113 (1987).

11. Cai, M. Influence of intermediate principal stress on rock fracturing and strength near excavation boundaries-Insight from numerical modeling. Int. J. Rock Mech. Min. Sci. 45, 763-772 (2008).

12. Pan, P.Z., Feng, X.T. \& Hudson, J. A. The influence of the intermediate principal stress on rock failure behaviour: A numerical study. Eng. Geol. 124, 109118 (2012).

13. Haimson, B. True Triaxial Stresses and the Brittle Fracture of Rock. Pure Appl. Geophys. 163, 11011130 (2006).
14. Fjær, E. \& Ruistuen, H. Impact of the intermediate principal stress on the strength of heterogeneous rock. J. Geophys. Res. Solid Earth 107, ECV-3 (2002).

15. Takahashi, M. \& Koide, H. Effect of the intermediate principal stress on strength and deformation behavior of sedimentary rocks at the depth shallower than $2000 \mathrm{~m}$. in ISRM international symposium (International Society for Rock Mechanics, 1989).

16. Park, K.H., Tontavanich, B. \& Lee, J.-G. A simple procedure for ground response curve of circular tunnel in elastic-strain softening rock masses. Tunn. Undergr. Space Technol. 23, 151-159 (2008).

17. Drucker, D. C. Some implications of work hardening and ideal plasticity. Q. Appl. Math. 7, 411-418 (1950).

18. Murrell, S. A. F. A criterion for brittle fracture of rocks and concrete under triaxial stress and the effect of pore pressure on the criterion. Rock Mech. 563-577 (1963).

19. Deng, S., Zheng, Y. \& Yue, C. Strength model and mesoscopic mechanism of intermediate principal stress effect on rock strength. Sci. Sin. Technol. 47, 306-315 (2017).

20. Zheng, Y. \& Deng, S. Failure probability model considering the effect of intermediate principal stress on rock strength. Math. Probl. Eng. 2015, (2015).

21. Cai, M. Rock mechanics and engineering(Second Edition). (Science Press, 2017).

22. Beer, F. P., Jr, E. R. J., DeWolf, J. T. \& Mazurek, D. F. Mechanics of Materials, 7th Edition. (McGrawHill Education, 2014).

23. CHEN, L., PENG, J., FAN, W. \& Sun, P. Analysis of surrounding rock mass plastic zone of round tunnel under non-uniform stress field based on the unified strength theory [J]. J. China Coal Soc. 1, 003 (2007). 